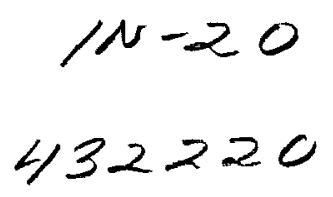

Lee M. Zook, ${ }^{\text {I }}$

\title{
Cathodic Protection Deployment on Space Shuttle Solid Rocket Boosters
}

\author{
REFERENCE: Zook, L. M., "Cathodic Protection Deployment on \\ Space Shuttle Solid Rocket Boosters," Designing Cathodic Protection \\ Systems for Marine Structures and Vehicles, ASTM STP 1370, H. P. Hack, \\ Ed., American Society for Testing and Materials, West Conshohocken, \\ PA, 1999.
}

\begin{abstract}
Corrosion protection of the space shuttle solid rocket boosters incorporates the use of cathodic protection(anodes) in concert with several coatings systems. The SRB design has large carbon/carbon composites(motor nozzle) electrically connected to an aluminum alloy structure. Early in the STS program, the aluminum structures incurred tremendous corrosive attack due primarily to the galvanic couple to the carbon/carbon nozzle at coating damage locations. Also contributing to the galvanic corrosion problem were stainless steel and titanium alloy components housed within the aluminum structures and electrically connected to the aluminum structures. This paper will highlight the evolution in the protection of the aluminum structures, providing historical information and summary data from the operation of the corrosion protection systems. Also, data and information will be included regarding the evaluation and deployment of inorganic zinc rich primers as anode area on the aluminum structures.
\end{abstract}

KEYWORDS: cathodic protection, aluminum, airframes, anode

\section{Background}

- With the launch of the Space Shuttle Columbia in 1981 NASA entered into a new paradigm of reusing space flight hardware. One of the major challenges among hardware designated for reuse was the Space Shuttle and the solid rocket boosters(SRB). While the space shuttle would land on a runway, like an airplane, the SRB was not so fortunate. The SRB landing occurs in the ocean at a velocity approaching $27 \mathrm{~m} / \mathrm{s}$. The SRB is towed through the ocean to a slip where it is removed from the water. This process can take between 24 and 72 hours depending on launch time and weather conditions. Figure 1 shows the SRB major components. The structure of greatest interest(where the greatest corrosion problems have occurred) is the aft skirt(figure 1).

\footnotetext{
${ }^{\mathrm{l}}$ Senior Materials Engineer, USBI Co., P.O. Box 21212, Kennedy Space Center, FL 32815
} 


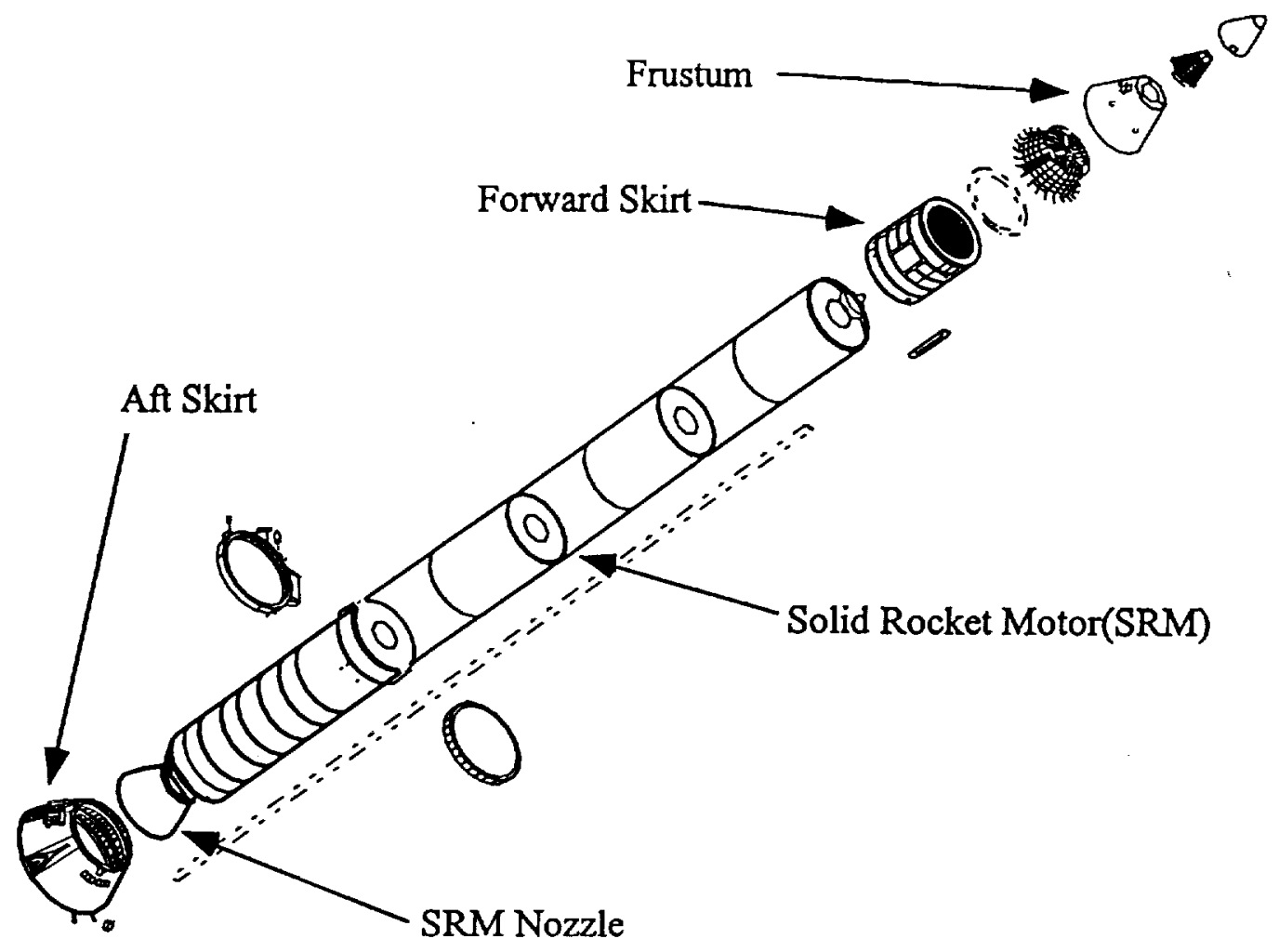

Figure 1-Solid Rocket Booster(SRB) exploded view.

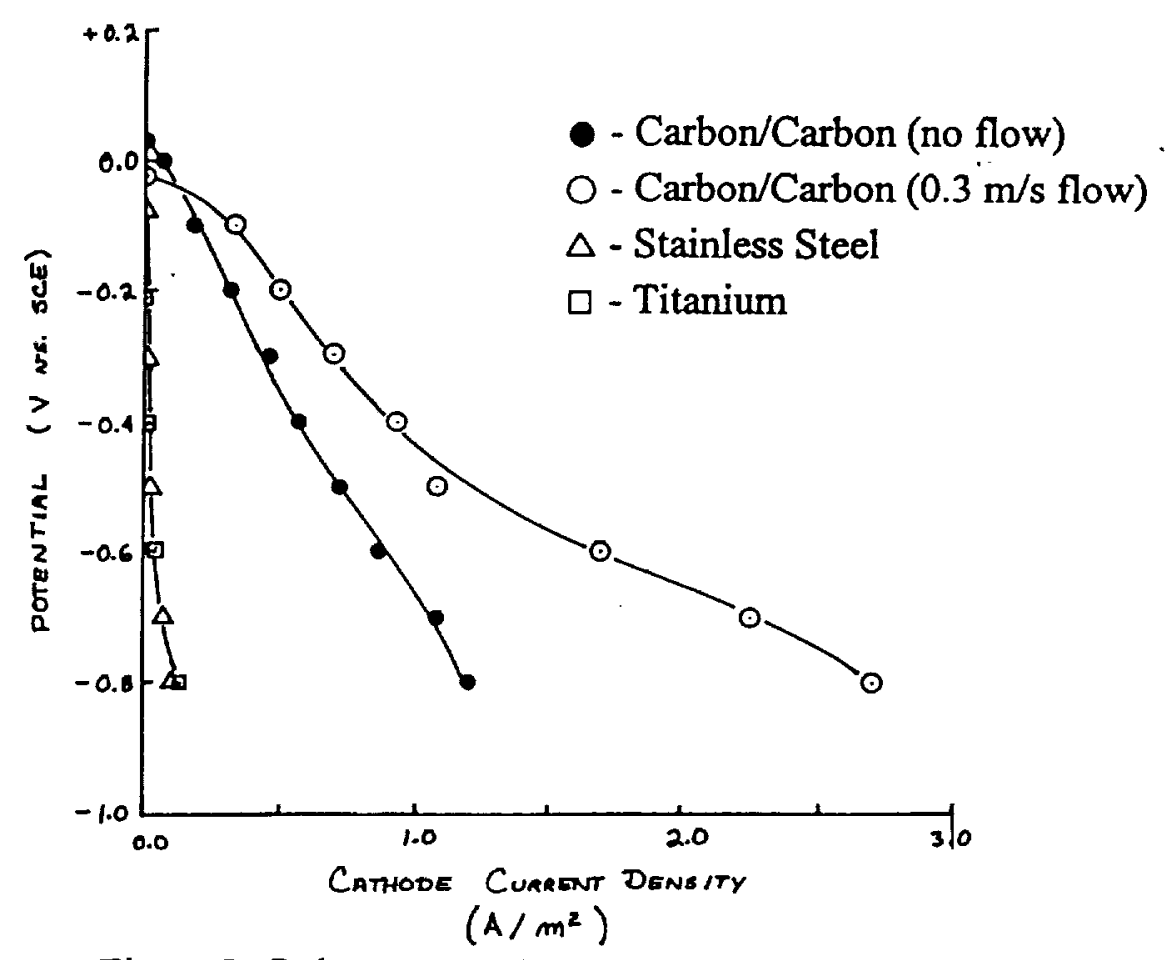

Figure 2-Polarization of cathode materials. 


\section{SRB Design}

The large scale reuse of space flight hardware was begun with the shuttle program. With regard to the SRB, NASA had little experience with the effects of the descent, splashdown and tow back environments on the hardware. The SRB aft skirt structure is weld constructed of aluminum alloy(AA) 2219-T87 with bolted in AA 2219-T87 reinforcements. Housed within the aft skirt is the thrust vector system(TVC). The TVC system provides the direction control for the solid rocket motor(SRM) nozzle. The TVC system is constructed of many alloy types including stainless steels, titanium and Inconel. Most of these alloys are left uncoated(bare). Table 1 lists the TVC system alloys which provide the majority of the cathode surface areas which are not coated. The SRM cases are a HSLA steel(painted) while the nozzle has a carbon/carbon liner bonded to a steel structure(painted). All components are electrically bonded for lightening protection grounding. On the exterior of the aft skirt a thermal protective coating is applied to protect the structure from any thermal loads during ascent. To protect the aft skirt interior and TVC system from radiant heating, a thermal blanket made from quartz glass and fiberglass is attached between the aft skirt and SRM nozzle.

Table 1-Summary of TVC System Exposed Surface Areas.

\begin{tabular}{cc}
\hline Alloy & Exposed Surface Area(m²) \\
\hline Titanium, Ti6A14V & 2.9 \\
Austenitic Stainless Steel & 2.5 \\
Inconel 625, 718 & 0.8 \\
Haynes 188 & 0.4 \\
17-4 PH & 0.1 \\
Nitronic 40 & 0.1 \\
\hline
\end{tabular}

The original corrosion protection system for the aluminum components consisted of a chromate conversion coating surface treatment, epoxy primer and epoxy topcoat. Bolted joints are sealed with a 2 component polysulfide sealant and all fasteners were oversealed. The original evaluation of corrosion protection materials performed by NASA included limited galvanic evaluations of the coating system with the TVC system alloys[1]. The results indicated that the coatings would provide good corrosion performance in galvanic contact. It should be noted that the coupons used for these evaluations were painted without being scribed or intentionally damaged. The original coating system was recently replaced with a chromate conversion coating, barium chromate epoxy primer and polyurethane topcoat. A significant design change which had a positive affect on the aft skirt corrosion was the addition of polyurethane foam to the interior surfaces(except behind the TVC system) of the aft skirt on flight STS-5 $(11 / 11 / 82)$.

\section{Initial Flight Results}

The corrosion which resulted from the first flights of the SRB exceeded most expectations. The corrosion primarily occurred at locations where the coatings had been 


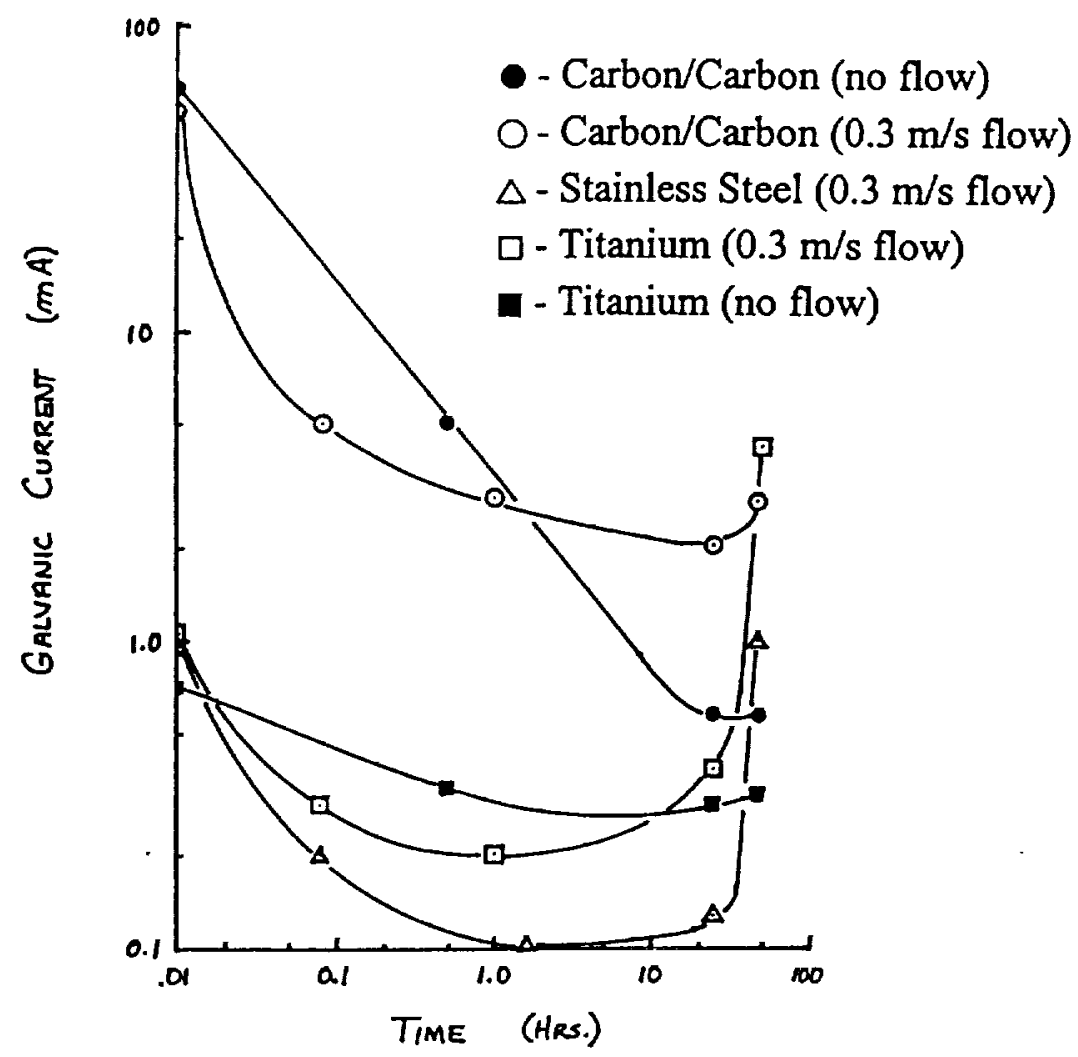

Figure 3- Galvanic couple currents for AA 2219 during a 48 hour exposure period.

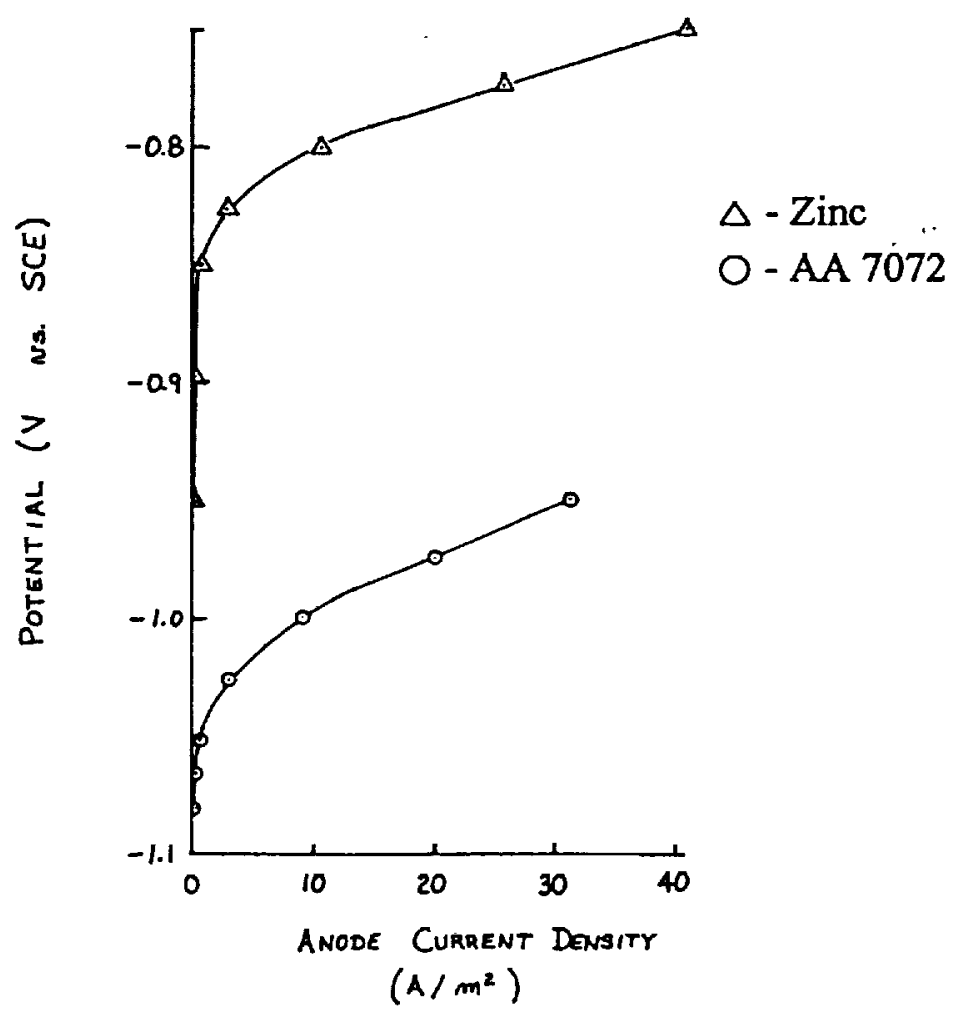

Figure 4- Polarization of anode materials $(0.3 \mathrm{~m} / \mathrm{s}$ flow). 
damaged during the descent and splashdown of the SRB. Damage sources included propellant slag, thermal blanket debris, water impact force and SRM exhaust hot gases. Also noted as contributing to the problem was poor coating application technique. A committee was formed to formally evaluate the postflight condition of the hardware and provide recommendations for effective corrosion control activities. Problems noted during the investigation included galvanic $\&$ crevice corrosion, coating damage during descent and a great number of workmanship issues. The committee provided recommendations which included addressing the galvanic, pitting and crevice corrosion situations. These recommendations provided the foundation for subsequent corrosion control and cathodic protection activities for the aft skirt structure.

\section{SRB Cathodic Protection System Design}

The approach for cathodic protection focused upon the use of sacrificial anodes in lue of impressed current systems. This was due to the relative simplicity of anodes and that anodes could be deployed with minimal flight hardware design changes. Initial anode system design focused upon understanding the strengths of the cathodic materials, evaluating anode alloys, determination of anode effectiveness under a special foam coating and developing a systematic approach to reduce the overall galvanic damage to the aluminum aft skirt structure. To address the first 3 issues, a series of galvanic experiments were conducted with the alloy being protected(AA 2219), the primary cathode areas(18-8 stainless steel, Ti6Al4V titanium alloy and carbon/carbon phenolic composite) and the anode candidates(zinc and AA 7072). The experiments conducted included polarization of the cathode and candidate anode materials and a determination of the relative strength of the anode materials for reducing the potential of the primary cathode areas.

\section{Experimental Results}

The testing confirmed that the carbon/carbon material was the greatest contributor to the galvanic problems(figure 2) and that a great amount of current would be required to polarize this material to reach the potential of the $2219 \mathrm{AA}(-0.82 \mathrm{~V}$ vs. Saturated calomel electrode(SCE)[2]). A surprising result was that flowing sea water on the carbon/carbon material more than doubled the current required to polarize the cathode to $-0.8 \mathrm{~V}$ (vs. SCE). Also, the stainless steel and titanium alloys would require very little current to be polarized to the same potential. In evaluating the galvanic currents of the primary cathode materials, it was determined(figure 3) that the carbon/carbon material had the greatest current in the first hour and then the current remained somewhat constant. The stainless steel and titanium cathodes current acted in a manner opposite to that of the carbon/carbon, in that their current remained somewhat constant initially but showed a significant increase around 48 hours of exposure.

The anode polarization data(figure 4) shows that the 7072 alloy would not be able to polarize the galvanic couples as easily as the zinc anode material. To determine the relative amount of anode area that would be required to polarize the respective cathode surface the amount of anode area was varied in relation to the cathode area(figure 5). 

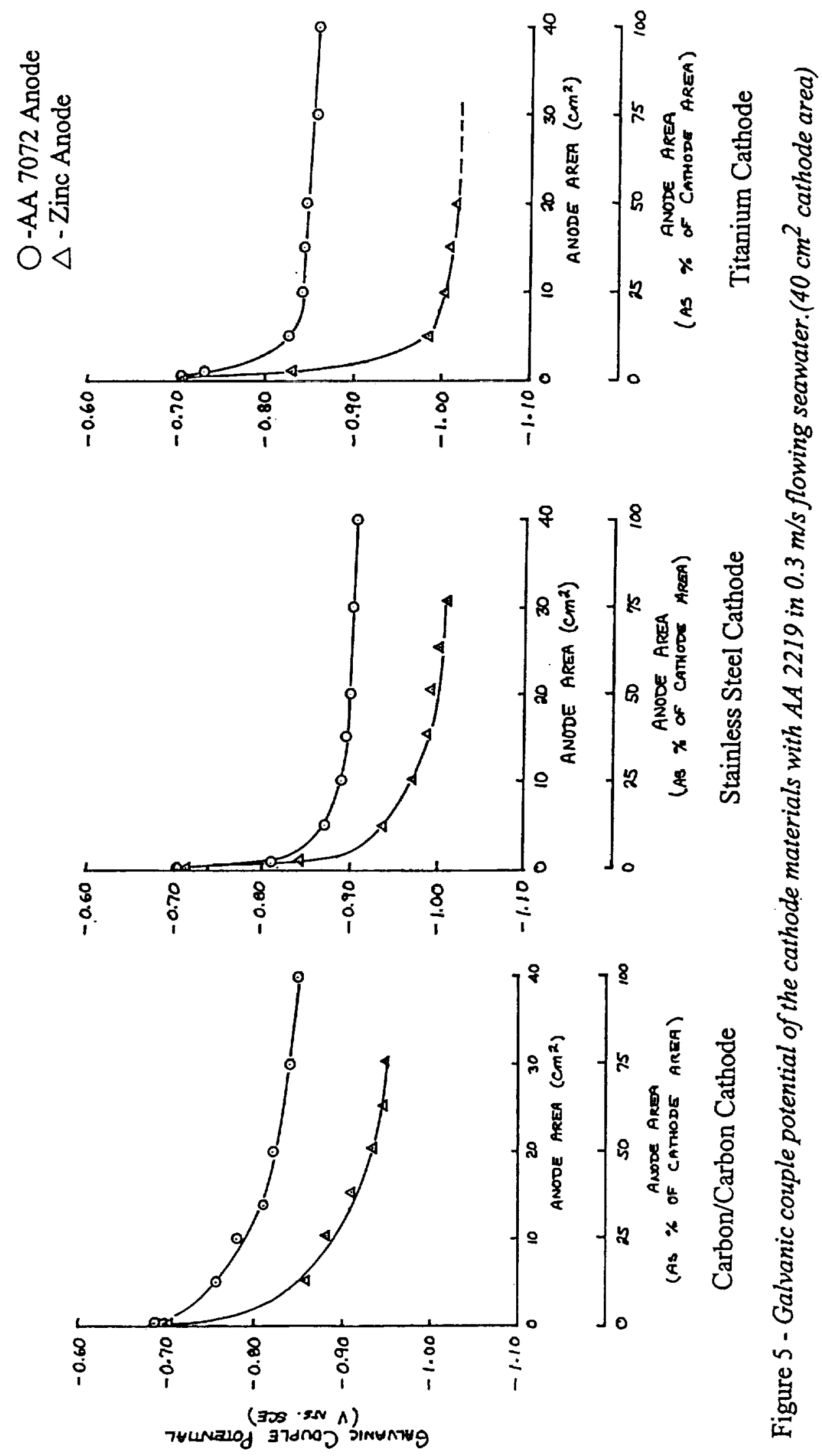
From these results(figure 5) it was decided that for the stainless steel and titanium surfaces the anode area should be about $20-25 \%$ of the cathode area and for the carbon/carbon material the anode area should be about $30-40 \%$ of the cathode area. Additional anode area beyond these percentages would provide minimal benefit to the aluminum protection. Testing of anode performance under the polyurethane foam indicated that once the foam was saturated with water the anodes performed normally. However, the length of time required to obtain water saturation could vary. Due to the unknown water saturation rate of the foam, it was decided not to deploy anodes under foam.

\section{Protection Approach}

The original plan for the corrosion protection of the aft skirts included the incorporation of zinc anode area, coating of cathodic surface area and isolating the SRM nozzle. The exposed cathode areas within the aft skirt(including alloys listed in table 1) total to approximately $23 \mathrm{~m}^{2}$. Based upon the galvanic couple potential results(figure 5) it was planned to deploy a total of $6.5 \mathrm{~m}^{2}$ of zinc area to negate the cathode affects on the aluminum structure. This amount of zinc could be reduced as the cathode areas were coated(planned cathode area reduction of $5.4 \mathrm{~m}^{2}$ ) and when nozzle isolation was incorporated(planned cathode area reduction of $16.1 \mathrm{~m}^{2}$ ) into the design. The hardware areas targeted for anode deployment were within the TVC system and on the SRM nozzle. The goal was to achieve a galvanic potential of $-1.0 \mathrm{~V}$ vs. SCE on the aluminum structure and components.

\section{Protection System Implementation}

Anode deployment occurred over a two year period beginning with flight STS-6(April 4,1983 ) and completed with flight STS-23(April 21, 1985). The anode deployment schedule is shown in table 2 . The anode area was obtained through the use of solid zinc anodes and thermally applied(flame spray) zinc coatings. The initial location of anode deployment was on 2 TVC components. The next deployment of area was through the use of a diver installed anode(DIA). The anode is usually installed within four hours of SRB splash down. It should be noted that both of these area deployments were made prior to the testing program discussed under the System Design. The total deployed anode area was $3.09 \mathrm{~m}^{2}$. While additional surface areas were planned for thermally applied zinc, new post Challenger accident non-destructive evaluation requirements halted the implementation.

The other actions from the original plan, the coating of cathodic surface area and isolating the SRM nozzle, were pursued with little success. The application of coatings to cathodic areas was met with tremendous resistance from the design engineering and operations organizations. They believed that since the cathodic components would not corrode they did not need to be "painted". They could not be convinced of the benefits of coating the cathode areas and stopped this part of the plan. Regarding the isolation of the SRM nozzle, several meetings were held with representatives of Thiokol Corporation(contractor for the SRM). The result of the meetings were that there were 
several paths which provided electrical grounding and that it would require a major redesign of the nozzle to motorcase interface to allow for severing the electrical ground. This aspect of the plan was halted. One positive item which came out of the meetings was that a more accurate calculation of the active carbon/carbon nozzle liner was obtained from the Thiokol engineers. Based upon the nozzle design, they determined the active area to be approximately $5.2 \mathrm{~m}^{2}$ as opposed to the $16.1 \mathrm{~m}^{2}$ area originally calculated.

Table 2-SRB Anode Deployment Schedule.

\begin{tabular}{ccc}
\hline Deployment Date / Flight & Location & Anode Surface Area $\left(\mathrm{m}^{2}\right)$ \\
\hline April 4, 1983 / STS-6 & TVC System-components & 0.13 \\
August 30, 1983 / STS-8 & Diver attached to aft skirt HDP & 0.61 \\
April 6, 1984 / STS-13 & DOP & 0.35 \\
November 8, 1984 / STS-19 & Nozzle-Thermal Curtain & 0.32 \\
& Brackets & 1.03 \\
January 24, 1985 / STS-20 & TVC System-covers & 0.65 \\
April 21, 1985/STS-23 & Nozzle-Thermal Curtain & \\
& Brackets \\
\hline
\end{tabular}

\section{Data Collection}

To evaluate the effectiveness of the anodes, potential measurement surveys were conducted. The survey was performed once the SRB was in the Trident turn basin at the Cape Canaveral Air Force Station. This is usually between 24-48 hours after SRB splash down. Limited results from the potential surveys which were conducted during the anode deployment activities are presented in table 3.

Table 3-Galvanic Potential Measurements(-V vs. SCE).

\begin{tabular}{|c|c|c|c|c|c|c|c|c|}
\hline \multirow[b]{2}{*}{ Flight } & \multirow[b]{2}{*}{$\begin{array}{c}\text { Total } \\
\text { Anode } \\
\text { Area }\left(\mathrm{m}^{2}\right)\end{array}$} & \multicolumn{7}{|c|}{ Measurement Location } \\
\hline & & $\begin{array}{l}\text { Nozzle } \\
\text { carbon/ } \\
\text { carbon }\end{array}$ & $\begin{array}{l}\text { Aft Skirt } \\
\text { Interior } \\
\text { Structure }\end{array}$ & $\begin{array}{l}\text { TVC } \\
\text { Frame }\end{array}$ & $\begin{array}{c}\text { TVC } \\
\text { Exhaust } \\
\text { Duct }\end{array}$ & $\begin{array}{c}\text { Blast } \\
\text { Container }\end{array}$ & $\begin{array}{c}\text { Diver } \\
\text { Operated } \\
\text { Plug } \\
\end{array}$ & $\begin{array}{c}\text { Diver } \\
\text { Installed } \\
\text { Anode }\end{array}$ \\
\hline $\begin{array}{c}\text { STS-5 } \\
11 / 11 / 82\end{array}$ & 0 & - & 0.71 & - & 0.72 & 0.71 & - & 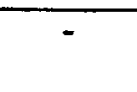 \\
\hline $\begin{array}{c}\text { STS-11 } \\
2 / 3 / 84\end{array}$ & 0.74 & 0.34 & 0.85 & - & 0.81 & 0.85 & - & 0.95 \\
\hline $\begin{array}{l}\text { STS-17 } \\
10 / 5 / 84\end{array}$ & 1.10 & 0.31 & 0.84 & 0.85 & 0.85 & 0.84 & 0.74 & 0.96 \\
\hline $\begin{array}{l}\text { STS-19 } \\
11 / 8 / 84\end{array}$ & 1.42 & 0.41 & 0.86 & 0.88 & 0.85 & 0.83 & 0.81 & 0.95 \\
\hline $\begin{array}{l}\text { STS-20 } \\
1 / 24 / 85\end{array}$ & 2.45 & 0.15 & 0.89 & 0.92 & 0.88 & - & 0.92 & 1.01 \\
\hline $\begin{array}{l}\text { STS-26 } \\
7 / 29 / 85\end{array}$ & 3.10 & 0.42 & 0.90 & 0.96 & 0.91 & 0.80 & 0.86 & 0.98 \\
\hline $\begin{array}{l}\text { STS-27 } \\
8 / 27 / 85\end{array}$ & 3.10 & 0.38 & 0.93 & 0.96 & 0.89 & 0.85 & 0.89 & 1.00 \\
\hline $\begin{array}{c}\text { STS-31 } \\
11 / 26 / 85\end{array}$ & 3.10 & 0.36 & 0.93 & 0.97 & 0.89 & 0.86 & 0.92 & 1.00 \\
\hline
\end{tabular}


As can be observed from the potential measurements, the aluminum structure potential has been shifted $-0.22 \mathrm{~V}$ from the pre-anode condition. Also, the aluminum TVC frame is almost at the $-1.0 \mathrm{~V}$ goal. Visual inspection of the aluminum components after removal from the water confirm that the anodes are performing well with minimal pitting observed at coating damage locations. While these results are good, several significant issues have arisen with the use of the anodes. The most significant is the desire to stop installing the DIA.

The use of a DIA has been controversial from the beginning of the effort to protect the aft skirt from corrosion. The use of the DIA was chosen because it was the quickest way to get anode area on to the SRB. During the cathodic protection design studies the galvanic current of the AA 2219 to cathode couples were evaluated(figure 3) with the finding that the cathode areas polarize quickly(within the first 4-5 hours). This would indicate that the zinc anode area needs to be available immediately upon water impact. The DIA is the last item installed on the SRB during recovery. Historically the DIA usually installed within 6.5 hours of water impact. However, there is no guarantee that the DAI will be installed at all(especially during rough seas). Recently, concerns of diver safety during the recovery operations have been raised and used as justification to plan for the elimination of the DIA. As a result of the DIA planned elimination there is a renewed interest in adding more anode area directly to the aft skirt structure.

\section{New Approaches to Anode Area}

The original plan developed for the aft skirt protection emphasized applying zinc by thermal spray directly to the TVC frames and aft skirt interior. Since the plan was approved, new constraints have been place upon these locations. Current postflight hardware evaluations includes the use of dye penetrant and ultrasonic nondestructive inspections. When several zinc coated TVC covers required NDE after being straightened, we found that removing the metallic zinc safely and quickly was very difficult. Since the zinc is not easily removed, the structural design group would not allow the application of metallic zinc directly to the structural components. As a result of this situation, two different approaches are being investigated to increase the anode areas.

To achieve the originally recommended $6.5 \mathrm{~m}^{2}$ of zinc surface area without enduring a significant weight penalty, it was conceptualized that an inorganic zinc rich primer(IZRP) could possibly provide the required protection. An additional concept that was recently introduced was to deploy anode area by using an expanded zinc(metal foam) product. Both of these approaches will be discussed in greater detail beginning with the IZRP.

\section{Inorganic Zinc Rich Primer Anode Area}

The advantages of using the primer included that it could be easily removed using conventional blasting techniques with plastic media, the primer could replace the coating system currently applied to the structures, the coating would offer better abrasion and heat resistance than the current coating system and that no special processes/equipment would be required to apply the IZRP to the hardware. While these advantages are important, several significant issues have to be addressed to assure that the hardware will 
be adequately protected. Issues raised included adhesion of the IZRP to aluminum, coating reuse and the anode performance of the coating. To determine the feasibility of the concept, limited adhesion and corrosion evaluations were performed.

AA 2219-T87 panels were prepared by cleaning the surface, abrasive blasting(anchor profile of $25-40 \mu \mathrm{m}$ ) or applying a pretreatment and applying a solvent borne, environmentally compliant IZRP to achieve a dry film thickness of approximately $75 \mu \mathrm{m}$. After completion of cure, pull off adhesion tests were performed. The results of these tests are shown in table 4. It should be noted that the zinc rich primer flaked off of the conversion coated surface prior to bonding anvils to the painted surface. This testing established that the IZRP could meet the minimum flight coating adhesion requirements of $4826 \mathrm{kPa}$.

\begin{tabular}{|c|c|}
\hline Surface Preparation Technique & Coating Adhesion(kPa) \\
\hline Chromate Conversion Coating & 0 \\
\hline glass bead(MIL-G-9954, \#6) blast & 5592 \\
\hline Aluminum oxide(20-30 mesh) blast & 6433 \\
\hline sodium bicarbonate blast & 7267 \\
\hline
\end{tabular}

The initial anode performance of the IZRP was assessed through the use of electrochemical impedance spectroscopy(EIS) and polarization resistance techniques. [3] The evaluation compared the performance of an epoxy zinc rich primer currently used on the SRB with a solvent based inorganic zinc rich primer. The results indicated that the IZRP coating would provide sufficient protection to the aft skirt, however, it was recommended that additional testing be performed to simulate the aft skirt use conditions.

\section{Metal Zinc Foam}

The concept of using a zinc foam for anode material came from work that was being performed using aluminum foam for energy absorption on the aft skirt hold down post frangible nuts. Discussions were held with the aluminum foam vendor to determine their ability to process zinc metal into foam. They reported that they have made zinc foam material for a battery company and was interested in our possible use of zinc foam material. They reported that with a foam density of 1.2 pores per $\mathrm{mm}$ it was possible to obtain $4.2 \mathrm{~m}^{2}$ surface area with a volume of $0.23 \mathrm{~m}^{3}$ and weight of $3.2 \mathrm{Kg}$. Samples are being obtained to further evaluate the performance of this anode material.

\section{Summary}

The as-implemented cathodic protection system has performed well in actual use. While the deployed anode area is approximately $50 \%$ of the original recommendation, no signs of aggressive corrosive attack has been observed in damaged coating locations. The disparity between theory and real life may be explained by the fact that the cathode areas were calculated on a worst case basis-all carbon/carbon nozzle liner completely intact and 
active. In reality, the splash down/water impact loads tend to cause flexing of the nozzle and debonding of the carbon/carbon material. The only planned improvement of the cathodic protection system will occur in conjunction with the deletion of the DIA. While the DIA accounts for $20 \%$ of the deployed anode area, the planned new area will attempt to fully implement the original recommendation of $6.5 \mathrm{~m}^{2}$ total anode area. Evaluation of both the IZRP and zinc metal foam solutions are underway and will hopefully be ready for deployment before the DIA is no longer installed.

\section{References}

[1] "SRB Materials and Processes Assessment From Laboratory and Ocean Environmental Tests", NASA TM-78187, National Aeronautics and Space

Administration, Washington, DC, 1978. (Public Domain LhP.)

[2] Hollingsworth, E. H. and Hunsicker, H. Y., "Corrosion of Aluminum and Aluminum Alloys," Metals Handbook 9th Edition, Volume 13 Corrosion, ASM International, Metals Park, OH, 1987, p 584.

[3] Danford, M. D., Walsh, D. W., and Mendrek, M. J., "The Corrosion Protection of 2219-T87 Aluminum by Organic and Inorganic Zinc-Rich Primers,"NASA TP-3534, National Aeronautics and Space Administration, Washington, DC, 1995 